\title{
miR-542-3p inhibits the growth and invasion of colorectal cancer cells through targeted regulation of cortactin
}

\author{
HAO-CHENG LONG ${ }^{1 *}$, XIA GAO ${ }^{2 *}$, CHANG-JIANG LEI ${ }^{1 *}$, BIN ZHU $^{1}$, LEI LI ${ }^{1}$, \\ CHENG ZENG $^{1}$, JIAN-BIN HUANG ${ }^{1}$ and JIA-RUI FENG ${ }^{1}$
}

Departments of ${ }^{1}$ General Surgery and ${ }^{2}$ Oncology, The Fifth Hospital of Wuhan, Wuhan, Hubei 430050, P.R. China

Received May 16, 2015; Accepted February 8, 2016

DOI: $10.3892 / \mathrm{ijmm} .2016 .2505$

\begin{abstract}
Colorectal cancer is one of the most common malignancies. Previous studies have reported that cortactin $(C T T N)$ is often overexpressed in tumors and is associated with metastasis and poor prognosis of patients. The abnormal expression of microRNAs (miRNAs or miRs) is closely related to the development and progression of various types of cancer, including colorectal cancer. However, little is known about the miRNAs targeting cortactin. In the present study, prediction using biological software revealed that cortactin has binding sites for miR-542-3p. Transfection with miR-542-3p mimic demonstrated that $\mathrm{miR}-542-3 \mathrm{p}$ reduced the expression of cortactin in colorectal cancer cells. Dual luciferase reporter assays further demonstrated that miR-542-3p regulated cortactin in a targeted manner and that miR-542-3p expression was significantly downregulated in colorectal cancer cells. A cell proliferation assay and Transwell migration assay were undertaken: we noted that miR-542-3p inhibited the proliferation and invasion of colorectal cancer cells while promoting their apoptosis. By contrast, cortactin acted antagonistically. When co-transfected with miR-542-3p mimic and $C T T N$ overexpression vector, the inhibitory effect of miR$542-3 p$ was blocked. This indicates that miR-542-3p regulates CTTN in a targeted manner to modulate the growth and invasion of colorectal cancer cells. The present study thus provides new targets for the prevention and treatment of colorectal cancer.
\end{abstract}

\section{Introduction}

Colorectal cancer is one of the most common malignancies, and its incidence has increased worldwide over the past two decades; it is the third most common cancer in the world (1). In China, it is the fourth most common cause of mortality (2).

Correspondence to: Dr Hao-Cheng Long, Department of General Surgery, The Fifth Hospital of Wuhan, 122 Xianzheng Street, Hanyang, Wuhan, Hubei 430050, P.R. China

E-mail: changjiang0118@163.com

${ }^{*}$ Contributed equally

Key words: miR-542-3p, cortactin, colorectal cancer, microRNA, CTTN
There is, therefore, an urgent need to study and understand the biological characteristics of colorectal cancer.

Cortactin is an actin-binding protein that is directly involved in the assembly of actin cytoskeleton. It was first identified as a substrate of oncogenic tyrosine kinase v-Src (3). Cortactin is encoded by the cortactin $(C T T N)$ gene (formerly known as EMS1), which is located on chromosome 11q13; this region is amplified in various types of cancer, including head and neck, ovarian, breast, liver, and lung cancer (4). Current data has revealed that cortactin is overexpressed in many human tumors, including head and neck cancer, esophageal squamous cell carcinoma, colon cancer, gastric cancer, hepatocellular carcinoma, breast cancer and ovarian cancer (5-8). Previous studies have reported that gene amplification and increased mRNA expression of cortactin are associated with the metastasis and poor prognosis of tumors (8). A previous study compared the expression profiles of 39 metastasis-related genes, including CTTN, in highly and poorly metastatic liver cancer cells (9). The results showed that cortactin overexpression in poorly metastatic liver cancer cells increased the metastatic ability of cancer cells and promoted the development of metastases (9). Several studies have also demonstrated that cortactin overexpression stimulates cell migration and metastasis in both aberrant cell lines and tumor cells $(8,10,11)$. Cortactin plays an important role in cytoskeleton remodeling. It modulates cell motility, invasiveness, synaptogenesis, phagocytosis, tumorigenesis and metastasis (12). Moreover, it also functions as a key component of the signaling pathway involved in cytoskeleton remodeling.

MicroRNAs (miRNAs or miRs) are a class of small, noncoding RNA molecules consisting of 18-25 nucleotides. They regulate the expression of target genes at the post-transcriptional level and are involved in various biological processes, such as cell proliferation, differentiation, apoptosis, metabolism and tumorigenesis (13). Although the exact mechanism and functions of miRNAs have not yet been fully characterized, experimental $(14,15)$ and clinical studies $(16,17)$ have reported that abnormal miRNA expression is involved in the development and progression of various cancers, including colorectal cancer. Currently, the specific expression of more than 100 miRNAs has been studied in colon cancer tissues, cell lines and normal tissues (18). miRNAs play an important role not only in the development of colon cancer, but also in its progression and metastasis; according to a retrospective study by the University of Tokyo, high miR-21 expression is associated with vascular 
invasion, liver metastasis and tumor staging. In addition, miR-155 expression was found to positively correlate with lymph node metastasis in colorectal cancer, and overexpression of these two miRNAs in colorectal cancer patients was linked with reduced survival (19). However, the role of cortactin and related miRNAs in colorectal cancer had not previously received much scholarly attention. With this in mind, the present study was undertaken to examine their important role in colorectal cancer.

\section{Materials and methods}

Cell culture. The human colorectal cancer cell lines, LoVo, HCT116 and 293T cells were obtained from the American Type Culture Collection (ATCC, Manassas, VA, USA; www. ATCC.org) and cultured in high-glucose Dulbecco's modified Eagle's medium (DMEM) containing 10\% fetal bovine serum (FBS) (both from Gibco-BRL, Gaithersburg, MD, USA). The cells were maintained at $37^{\circ} \mathrm{C}$ in an atmosphere of humidified air with $5 \% \mathrm{CO}_{2}$ in a cell culture incubator.

Overexpression vector construction and transfection. RNA was extracted from LoVo cells using TRIzol (Invitrogen, Carlsbad, CA, USA) according to the manufacturer's instructions, followed by reverse transcription-quantitative polymerase chain reaction (RT-qPCR) to amplify the coding region of CTTN. The products were then digested with BamHI and EcoRI (Takara Biotechnology Co., Ltd., Dalian, China), cloned into pcDNA3.1 vectors, sequenced and verified. The primers used for CTTN amplification are shown in Table I. We used the biological algorithms, miRBase, miRanda, PITA and RegRNA, to predict candidate miRNAs in the 3'UTR region of the CTTN gene. Hsa-miR-542-3p mimic and mimic negative controls were synthesized by Shanghai GenePharma Co., Ltd. (Shanghai, China), and cortactin siRNA was purchased from Santa Cruz Biotechnology, Inc. (Dallas, TX, USA). The cells were seeded onto a 6 -well plate at $1 \times 10^{5}$ cells $/ \mathrm{ml}$ and incubated for $24 \mathrm{~h}$. Transfection was performed using Lipofectamine 2000 (Invitrogen) according to the manufacturer's instructions, when cell confluence reached approximately $70 \%$. The concentrations of plasmid, miRNA and siRNA for transfection were $4 \mu \mathrm{g} / \mathrm{ml}, 100 \mathrm{nM} /$ well and $100 \mathrm{nM} /$ well, respectively.

$R T-q P C R$. Total RNA was extracted from the cells using TRIzol (Invitrogen). One microgram of total RNA was used for the firststrand cDNA synthesis with a RevertAid ${ }^{\mathrm{TM}}$ First Strand cDNA Synthesis kit (Fermentas GmbH, St. Leon-Rot, Germany). RT-qPCR was performed using $10 \mu \mathrm{l} 2 \mathrm{X}$ SYBR-Green PCR Master Mix (Toyobo, Tokyo, Japan), with $5 \mu \mathrm{l}$ cDNA, $0.5 \mu \mathrm{l}$ forward primer, $0.5 \mu 1$ reverse primer, and $4 \mu \mathrm{l}$ RNase-free $\mathrm{ddH}_{2} \mathrm{O}$ contained in $20 \mu \mathrm{l}$ of reaction mixture. The primers used for CTTN amplification are shown in Table I. The reaction was performed as follows: one cycle of $95^{\circ} \mathrm{C}$ for $5 \mathrm{~min}$ and 40 cycles of $95^{\circ} \mathrm{C}$ for $30 \mathrm{sec}, 58^{\circ} \mathrm{C}$ for $30 \mathrm{sec}$ and $72^{\circ} \mathrm{C}$ for $30 \mathrm{sec}$. Three independent experiments were conducted for each sample. Data were compared using the $2^{-\Delta \Delta \mathrm{Ct}}$ method.

Western blot analysis. Total cellular proteins were extracted by incubating the cells in lysis buffer [1X PBS, $1 \%$ NP-40, $0.1 \%$ sodium dodecyl sulfate (SDS), $5 \mathrm{mM}$ ethylenediaminetetraacetic acid (EDTA), $0.5 \%$ sodium deoxycholate, and $1 \mathrm{mM}$ sodium with protease inhibitors]. The protein concentrations in the cell lysates were determined by bicinchoninic acid assay (Pierce, Rockford, IL, USA). SDS-polyacrylamide gel electrophoresis (PAGE) was performed in $8 \%$ glycine gels (Bio-Rad, Berkeley, CA, USA) loading equal amounts of proteins per lane. Following electrophoresis, the separated proteins were transferred to nitrocellulose membranes (Pierce) and blocked with 5\% non-fat milk in TBST buffer for $1 \mathrm{~h}$. The membranes were then incubated with cortactin (polyclonal, rabbit, \#3502) and glyceraldehyde 3-phosphate dehydrogenase (GAPDH; monoclonal, rabbit, \#3683) antibodies (Cell Signaling Technology, Inc., Danvers, MA, USA) at 1:1,000 dilutions in $5 \%$ non-fat milk overnight at $4^{\circ} \mathrm{C}$, and then anti-rabbit $\mathrm{IgG}$ monoclonal antibody conjugated with horseradish peroxidase (\#7074; Cell Signaling Technology, Inc.) at 1:2,000 dilution for $1 \mathrm{~h}$ at room temperature. Protein bands were detected using the West Femto system (Pierce).

Dual luciferase assay. The CTTN 3'UTR was amplified from cDNA (primers are shown in Table I) and cloned into a psiCHECK-2 vector (Promega Corp., Beijing, China). A QuikChange II Site-Directed Mutagenesis kit (Stratagene, La Jolla, CA, USA) was used in order to generate mutant-type CTTN 3'UTR in which the seven mutated nucleotides were underlined within the seed region of the miR-542-3p binding site. A reporter vector psiCHECK-2 carrying the 3'UTR sequences of CTTN was assayed for luciferase expression using the Dual-Glo Luciferase assay system (Promega Corp.) according to the manufacturer's instructions. Three independent experiments were performed.

Cell proliferation. Cell proliferation was estimated in 96-well plates using a colorimetric immunoassay, based on the measurement of 5-bromo-2'-deoxyuridine (BrdU) incorporation during DNA synthesis (BrdU ELISA kit; Roche Diagnostics GmbH, Mannheim, Germany). Following miRNA and/or plasmid transfection for $48 \mathrm{~h}$, the medium was removed and cells were labeled with $\mathrm{BrdU}(10 \mathrm{mM})$ for $3 \mathrm{~h}$ at $37^{\circ} \mathrm{C}$. The cells were fixed and incubated with peroxidase-conjugated anti-BrdU antibody (sheep polyclonal, 11647229001) for $90 \mathrm{~min}$ at room temperature. The peroxidase substrate, 3,3',5,5'-tetramethylbenzidine, was then added, and BrdU incorporation was quantified by differences in absorbance at a wavelength of 370 minus $492 \mathrm{~nm}$. Cell proliferation was expressed as the mean percentage of the control values (set at 100\%).

Cell cycle analysis. The cells were pelleted (400 x g for $5 \mathrm{~min}$ at $4^{\circ} \mathrm{C}$ ), washed twice with cold PBS, resuspended in $500 \mu \mathrm{l}$ cold PBS, and then fixed for $1 \mathrm{~h}$ at $4^{\circ} \mathrm{C}$ by adding $500 \mu \mathrm{l}$ fixation solution ( $2 \% \mathrm{w} / \mathrm{v}$ paraformaldehyde in $\mathrm{PBS}, \mathrm{pH} 7.2)$. The fixed cells were pelleted, washed with cold PBS, resuspended in $1 \mathrm{ml}$ of $70 \%$ ethanol added dropwise to the pellet while vortexing and then incubated overnight at $4^{\circ} \mathrm{C}$. On the following day the cells were pelleted and resuspended in $1 \mathrm{ml}$ propidium iodide solution ( $40 \mu \mathrm{g} / \mathrm{ml}$ with $100 \mu \mathrm{g} / \mathrm{ml}$ RNase A) for $30 \mathrm{~min}$ at $37^{\circ} \mathrm{C}$ in the dark and analyzed using a FACScan flow cytometer (BD Biosciences, Franklin Lakes, NJ, USA).

Transwell Matrigel invasion assay. Invasion was evaluated using a Transwell Matrigel invasion assay (BD Biosciences, 
Table I. Sequences of primers.

\begin{tabular}{lllc}
\hline Name & \multicolumn{1}{c}{ Sense $\left(5^{\prime} \rightarrow 3^{\prime}\right)$} & \multicolumn{1}{c}{ Antisense $\left(5^{\prime} \rightarrow 3^{\prime}\right)$} & Product sizes $(\mathrm{bp})$ \\
\hline $\begin{array}{l}\text { Primer set for CTTN } \\
\text { CDS amplification }\end{array}$ & CGGGATCCGCCACCATGT & CG GAATTCCTACTGCC & \\
Primer set for RT-qPCR & & GCAGCTCCACAT & \\
GAPDH & GGTATCGTGGAAGGACTC & GTAGAGGCAGGGATGATG & 128 \\
CTTN & GCCGACCGAGTAGACAAG & GTATTTGCCGCCGAAACC & 102 \\
Primer set for CTTN & CCGCTCGAGAGCTGCGCCCT & ATAAGAATGCGGCCGCTCATA & \\
3'UTR amplification & GGATCCTCA & CCTATGAGGTGTGCTACAGG & \\
\hline
\end{tabular}

CDS, coding sequence; CTTN, cortactin. The underlining represents the restriction enzyme cutting site.
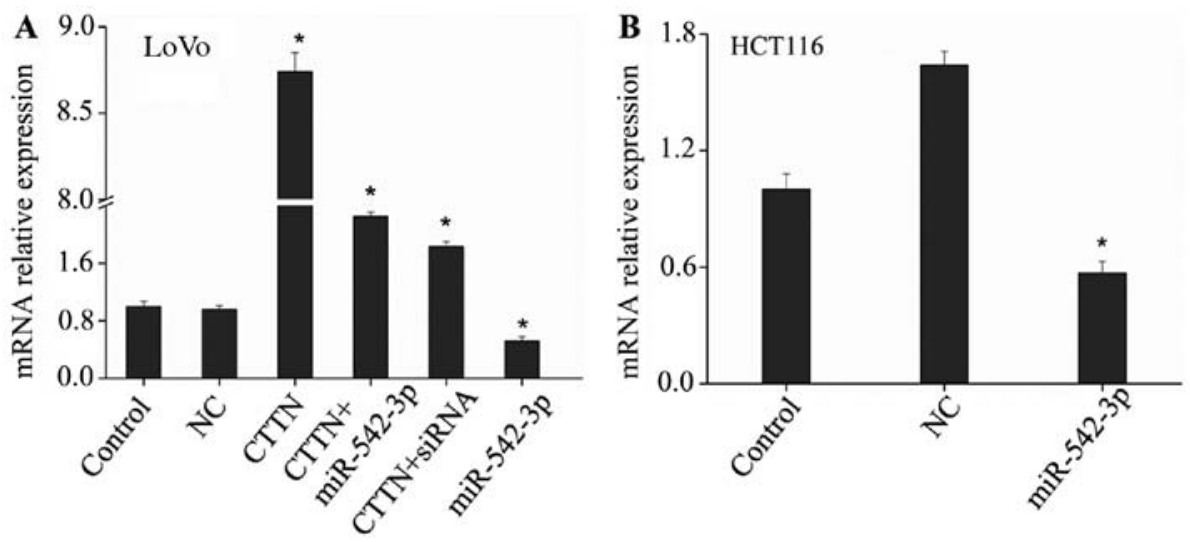

Figure 1. CTTN expression in LoVo and HCT116 cells. (A) LoVo and (B) HCT116 cells were transfected with miR-542-3p mimic and/or CTTN overexpression vector and then measured using RT-qPCR. The experiments were carried out at least in triplicate and the results are expressed as the means \pm SD ("P $<0.01$ vs. control). Control, untreated cells; NC, negative control.

Shanghai, China). Briefly, $200 \mu 1$ of the LoVO cells following transfection $\left(1 \times 10^{6}\right.$ cells $\left./ \mathrm{ml}\right)$ and $600 \mu 1$ complete medium were added to the upper and lower compartments of the chamber, respectively. Following incubation for $48 \mathrm{~h}$, the cells migrating to the lower side of the filter were fixed with $4 \%$ paraformaldehyde for $15 \mathrm{~min}$ at room temperature, washed with PBS, stained with crystal violet, and then observed under an inverted microscope (CKX41; Olympus Corp, Tokyo, Japan).

Statistical analysis. The experiments were performed at least in triplicate, and the results are expressed as the means \pm SD. Statistical analysis was performed using the SPSS statistical program version 17 (SPSS Inc., Chicago, IL, USA). A P-value $<0.01$ was considered to indicate a statistically significant difference.

\section{Results}

Prediction and validation of CTTN candidate miRNAs. In the present study, we used the biological algorithms miRBase, miRanda, PITA and RegRNA to predict candidate miRNAs in the 3 'UTR region of the $C T T N$ gene. In so doing, we identified hsa-miR-542-3p. Although previous studies have reported that miR-542-3p was downregulated in various types of tumor tissues and cells $(20,21)$, few studies have evaluated its functions. The mimic of the above miRNA was synthesized and transfected into the LoVo and HCT116 cells. RT-qPCR and western blot analysis proved that hsa-miR-542-3p inhibits the expression of CTTN (Fig. 1 and 2). This indicates that miR-542-3p regulates CTTN expression by targeting this gene. However, this finding warrants further validation by dual-luciferase reporter assays.

CTTN is a target gene of miR-542-3p. The 3'UTR region of the $C T T N$ gene was cloned into psiCHECK-2, a dual luciferase reporter vector, and the target sites of miR-542-3p were mutated (Fig. 3A, the underlined bases). The two plasmids were subsequently transfected, together with miR-542-3p mimic, into $293 \mathrm{~T}$ cells, and the changes in luciferase expression were analyzed. Luciferase expression in the cells transfected with plasmids containing the $3^{\prime} \mathrm{UTR}$ of $C T T N$ significantly declined by approximately $44 \%$ compared with the cells transfected with an empty reporter vector (Fig. 3B). By contrast, luciferase activity did not change significantly in the cells transfected with the plasmids containing the mutant 3'UTR of the CTTN gene. This indicates that miR-542-3p is strongly bound to the $3^{\prime}$ UTR of the $C T T N$ gene, suggesting that $C T T N$ is a target gene of miR-542-3p.

miR-542-3p affects colorectal cancer cell proliferation and apoptosis by targeting CTTN. BrdU assays were performed 

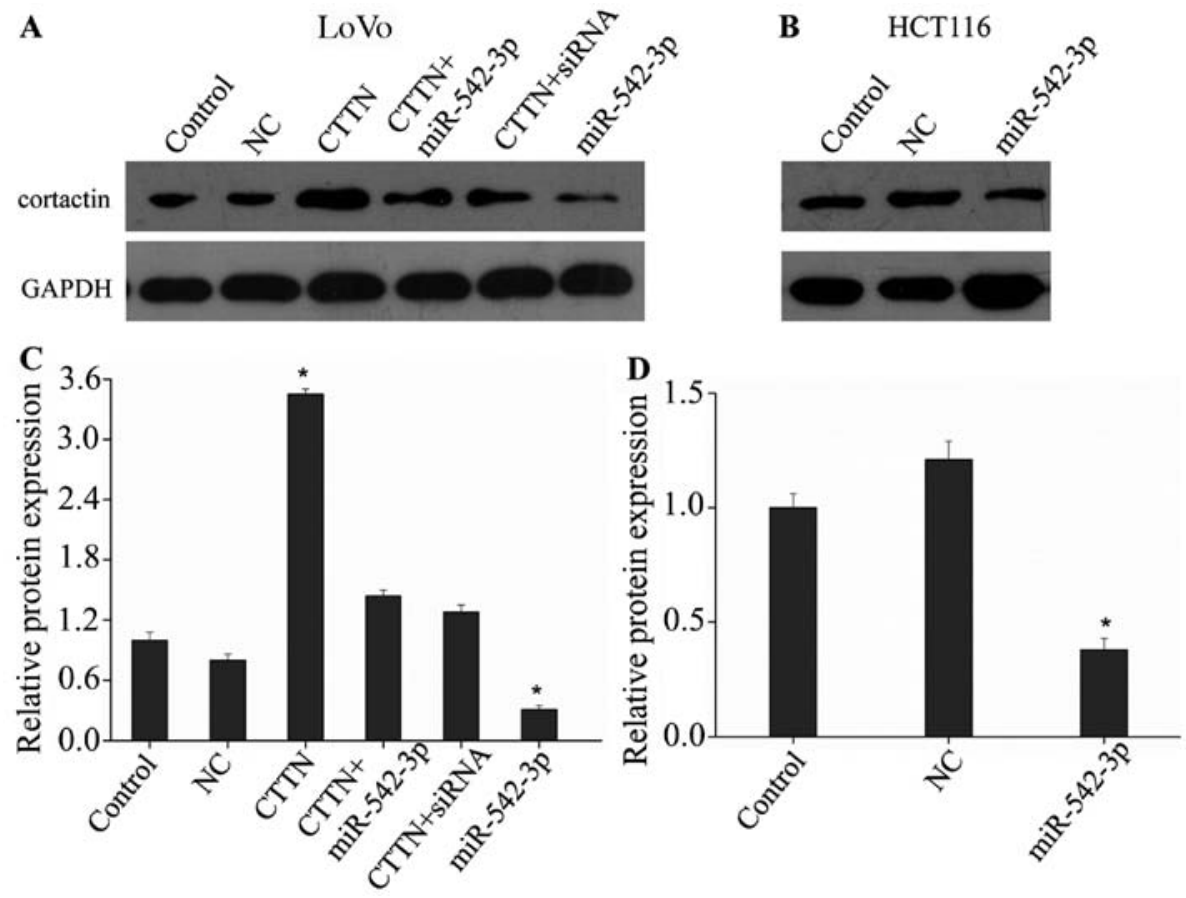

Figure 2. Cortactin (CCTN) expression in colorectal cancer cells. Cortactin expression in LoVo and HCT116 cells following transfection with miRNA mimic and/or CTTN overexpression vector, as measured using western blot analysis. Each bar represents the means \pm SD from three samples ("P $<0.01$ ). Control, untreated cells; $\mathrm{NC}$, negative control.

A miR-542-3p: 3' aaagucaaUAGUUAGACAGUGu 5'

CTTN wt 3' UTR: $\quad$ 5' uuugccaaAUUGA - CUGUCACg 3' $^{\prime}$

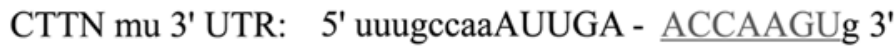

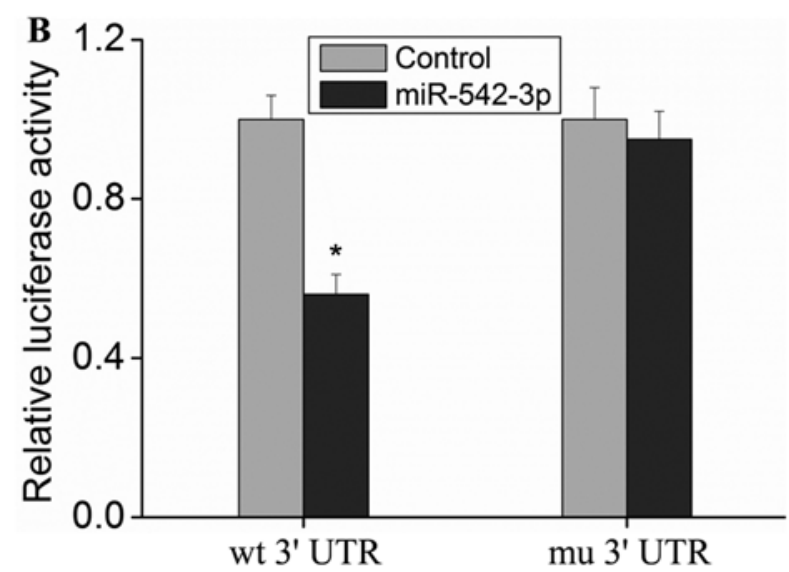

Figure 3. CTTN is a target gene of miR-542-3p. (A) miR-542-3p target sites in the sequence of cortactin (CCTN) 3'UTR. (B) The luciferase expression level of 293T cells transfected with cloning CTTN 3'UTR vector or mutant CTTN 3'UTR vector and miR-542-3p mimic. The values are expressed as the means \pm SD from three samples ( $\mathrm{P}<0.01$ vs. control). Control, cells transfected with empty reporter vector.

to evaluate the effects of increased miR-542-3p expression on human colorectal cancer cell proliferation. The results showed that miR-542-3p significantly inhibited the proliferation of colorectal cancer cells, whereas CTTN overexpression increased the proliferation of colorectal cancer cells (Fig. 4). Co-transfection with miR-542-3p mimic and the vector causing the overexpression of CTTN abolished the anti-proliferative role of miR-542-3p. Flow cytometric analysis of the cell cycle and apoptosis demonstrated that the percentage of cells in the sub-G1 peak increased significantly when LoVo cells were transfected with miR-542-3p mimic (Fig. 5). Co-transfection with the miR-542-3p mimic and the CTTN overexpression vector overcame the pro-apoptotic effect of miR-542-3p. Tumor cells are resistant to apoptosis; however, the increased expression of miR-542-3p promoted apoptosis of tumor cells, indicating that miR-542-3p inhibits tumor growth.

miR-542-3p inhibits the invasion of colorectal cancer cells by targeting CTTN. Tumor cells are known to be generally invasive and metastatic (22). To determine whether 

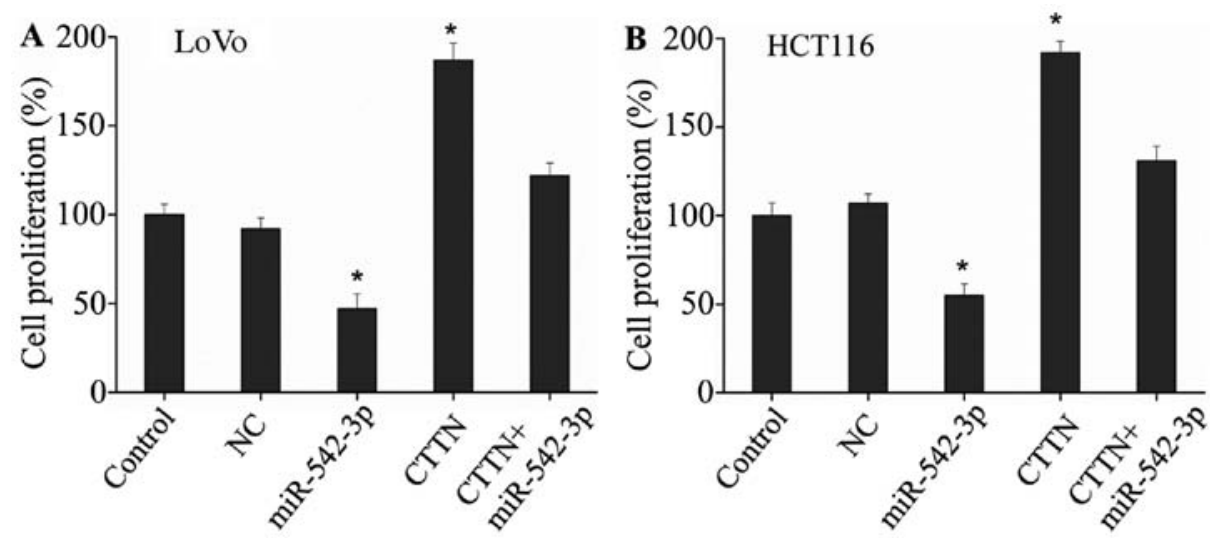

Figure 4. Effects of miR-542-3p and cortactin (CCTN) on colorectal cancer cell proliferation. The cells were transfected with miR-542-3p mimic and/or CTTN overexpression vector, and cell proliferation was examined using a BrdU assay. Each bar represents the means \pm SD from three samples ( $\left.{ }^{*}<0.01\right)$. Control, untreated cells; $\mathrm{NC}$, negative control.



Figure 5. Effects of miR-542-3p and cortactin (CCTN) on the cell cycle distribution of LoVo cells as examined by flow cytometric analysis. The images are representative of three independent experiments with similar results. Control, untreated cells; NC, negative control.

miR-542-3p inhibits the invasive ability of colorectal cancer cells, we performed Transwell migration assays. We found that an increase in miR-542-3p expression significantly inhibited the invasive ability of LoVo cells, while CTTN overexpression increased the invasion rate (Fig. 6). Co-transfection with miR-542-3p mimic and CTTN overexpression vector overcame the anti-invasive effect of miR-542-3p. This shows that miR-542-3p inhibits the invasion of colorectal cancer cells by targeting the CTTN gene.

\section{Discussion}

Colorectal cancer is known to be one of the most common malignancies; it is the third most common cancer and the second most deadly cancer. Its incidence and mortality are on the rise in China $(1,2)$. Invasion and metastasis are major causes of resultant mortality in patients with colorectal cancer (18). No effective solution is currently available for controlling colorectal metastases, and the prevention and treatment of colorectal cancer metastases has long been a challenging issue facing the medical community.

Previous research has reported that the invasion and the metastasis of malignant tumors, such as colorectal cancer, is a complex, multistep process. This complex process is possible as cancer cells are motile (23). Motility is an essential, common feature of various stages in the invasion and metastasis of cancer cells (24). Previous research has reported that during the movement of cancer cells, actin polymerization (assembly) is the power source of cancer cell motility (25). It has previously been shown that actin polymerization depends on the catalysis of the Arp 2/3 complex, and the activation of the Arp 2/3 complex requires the $C T T N$-encoded product, cortactin (26). Cortactin is expressed in colon cancer cells and plays an important role in the motility of cancer cells (27). Lee et al (5) have reported that the high expression of CTTN positively correlates with the depth of invasion and distant metastases of colorectal cancer. 

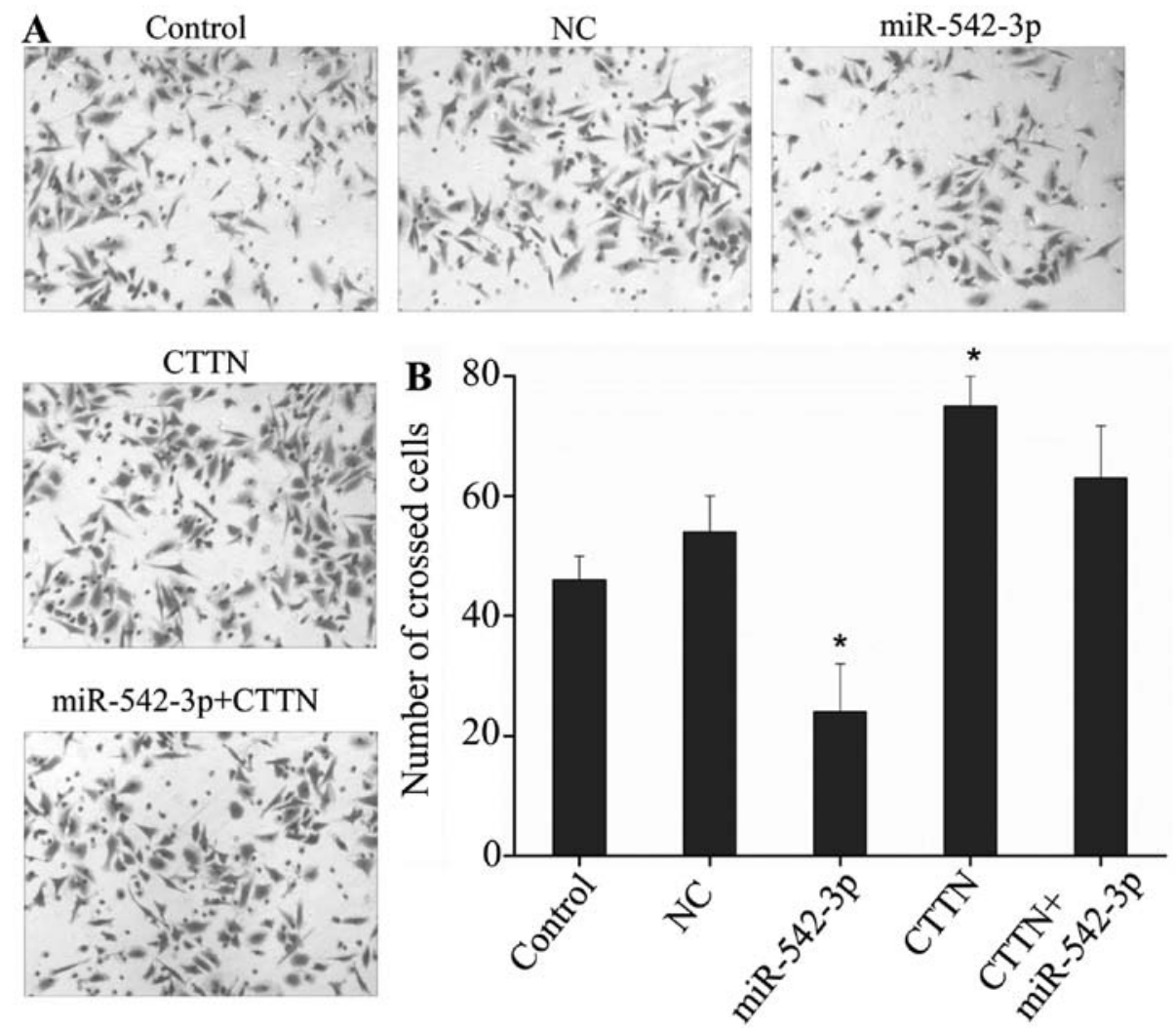

Figure 6. Effects of miR-542-3p and cortactin (CCTN) on the invasive ability of LoVo cells. (A) The cells were transfected with miR-542-3p mimic and/or CTTN-overexpressing vector, and the invasion of cells was evaluated using a Transwell Matrigel invasion assay. (B) Quantitative analysis of the anti-invasive effects of miR-542-3p and cortactin. Each bar represents the means \pm SD from 3 samples (" $\mathrm{P}<0.01$, vs. control). Control, untreated cells; NC, negative control.

Several studies have reported that the abnormal expression of miRNAs is closely related to the development, progression, and prognosis of colorectal cancer $(28,29)$. Notable progress has been made in the understanding of the biogenesis and mechanisms of action of miRNAs and their role in the diagnosis, treatment, and prognosis of colorectal cancer. However, few studies have been performed on miRNAs targeting CTTN. Only Zhang et al (30) found that miRNA-182 inhibits the proliferation and invasion of human lung adenocarcinoma cells through its effect on human cortical actin-associated protein. Hong et al (31) showed that VEGF-C increased CTTN expression by downregulating Dicer-mediated maturation of miR326, thereby relieving the suppressive effect of miR326 on CTTN expression. Given the important role of CTTN and miRNAs in colorectal cancer, we performed this study to gain greater insight into this issue. We found that miR-542-3p has target sites on CTTN. Moreover, the upregulation of miR-542-3p inhibited the expression of CTTN. Dual-luciferase reporter assays further confirmed that CTTN is a target gene of miR-542-3p.

A study by Wang et al (32) demonstrated that the circulating miRNAs, miR-646, miR-141 and miR-542-3p, potentially serve as non-invasive biomarkers for cervical squamous cell carcinoma. Althoff et al (20) concluded that miR-542-3p exerts its tumor suppressive function in neuroblastoma, at least in part, by targeting survivin and the expression of miR-542-3p, and thus targeting these is a promising therapeutic strategy for treating aggressive neuroblastoma. Thus, miR-542-3p has been demonstrated to function as a tumor suppressor gene, and to be involved in the development and progression of cancer.
However, its specific mechanism of action remains unclear. In the present study, we proved that miR-542-3p inhibits the proliferation and invasion of colorectal cancer cells, and promotes their apoptosis by targeting the CTTN gene. The results of a study by Yoon et al (21) suggested that survivin is a direct target of miR-542-3p and growth inhibition by miR-542-3p has potential application as an anti-cancer therapy. Wang et al (33) have found that overexpression of miR-542-3p in cancer cells elevated p53 expression, stimulated the expression of p53 targets, and inhibited cell proliferation. It has been demonstrated by $\mathrm{He}$ et al (34) that miRNA-542-3p inhibits tumor angiogenesis by targeting angiopoietin-2. Moreover, Shen et al (35) have previously suggested that miRNA-542-3p suppresses the cell growth of gastric cancer cells by targeting oncogene astrocyte-elevated gene-1. Thus, miR-542-3p functions as a tumor suppressor gene by inhibiting the proliferation and invasion of tumor cells.

In conclusion, miR-542-3p inhibits CTTN expression by targeting this gene in colorectal cancer cells and hence suppresses the proliferation and invasion, as well as promoting the apoptosis, of colorectal cancer cells.

\section{Acknowledgements}

This study was supported by the Wuhan City Health Bureau of Medical Research project (no. WX12C27), the Wuhan Municipal Science and Technology Bureau of Applied Basic research project (no. 2015061701011630), the Education Department of Hubei Province Science and Technology 
research project (no. B2015230), and the fourth batch of 'Hanyang Concert Talent Plan'.

\section{References}

1. Siegel R, Ma J, Zou Z and Jemal A: Cancer statistics, 2014. CA Cancer J Clin 64: 9-29, 2014.

2. Li M and Gu J: Changing patterns of colorectal cancer in China over a period of 20 years. World J Gastroenterol 11: 4685-4688, 2005.

3. Rothschild BL, Shim AH, Ammer AG, Kelley LC, Irby KB, Head JA, Chen L, Varella-Garcia M, Sacks PG, Frederick B, et al: Cortactin overexpression regulates actin-related protein $2 / 3$ complex activity, motility, and invasion in carcinomas with chromosome 11q13 amplification. Cancer Res 66: 8017-8025, 2006.

4. Schuuring E: The involvement of the chromosome 11q13 region in human malignancies: cyclin D1 and EMS1 are two new candidate oncogenes - a review. Gene 159: 83-96, 1995.

5. Lee YY, Yu CP, Lin CK, Nieh S, Hsu KF, Chiang H and Jin JS Expression of survivin and cortactin in colorectal adenocarcinoma: association with clinicopathological parameters. Dis Markers 26: 9-18, 2009.

6. Luo ML, Shen XM, Zhang Y, Wei F, Xu X, Cai Y, Zhang X, Sun YT, Zhan QM, Wu M and Wang MR: Amplification and overexpression of CTTN (EMS1) contribute to the metastasis of esophageal squamous cell carcinoma by promoting cell migration and anoikis resistance. Cancer Res 66: 11690-11699, 2006.

7. Timpson P, Wilson AS, Lehrbach GM, Sutherland RL, Musgrove EA and Daly RJ: Aberrant expression of cortactin in head and neck squamous cell carcinoma cells is associated with enhanced cell proliferation and resistance to the epidermal growth factor receptor inhibitor gefitinib. Cancer Res 67 9304-9314, 2007.

8. Buday L and Downward J: Roles of cortactin in tumor pathogenesis. Biochim Biophys Acta 1775: 263-273, 2007.

9. Chuma M, Sakamoto M, Yasuda J, Fujii G, Nakanishi K, Tsuchiya A, Ohta T, Asaka M and Hirohashi S: Overexpression of cortactin is involved in motility and metastasis of hepatocellular carcinoma. J Hepatol 41: 629-636, 2004.

10. Weaver AM: Cortactin in tumor invasiveness. Cancer Lett 265: 157-166, 2008.

11. van Rossum AG, Moolenaar WH and Schuuring E: Cortactin affects cell migration by regulating intercellular adhesion and cell spreading. Exp Cell Res 312: 1658-1670, 2006.

12. Ayala I, Baldassarre M, Giacchetti G, Caldieri G, Tetè S, Luini A and Buccione R: Multiple regulatory inputs converge on cortactin to control invadopodia biogenesis and extracellular matrix degradation. J Cell Sci 121: 369-378, 2008.

13. Esquela-Kerscher A and Slack FJ: Oncomirs - microRNAs with a role in cancer. Nat Rev Cancer 6: 259-269, 2006.

14. Hashimoto Y, Akiyama Y and Yuasa Y: Multiple-to-multiple relationships between microRNAs and target genes in gastric cancer. PLoS One 8: e62589, 2013.

15. Iwaya T, Yokobori T, Nishida N, Kogo R, Sudo T, Tanaka F, Shibata K, Sawada G, Takahashi Y, Ishibashi M, et al: Downregulation of miR-144 is associated with colorectal cancer progression via activation of mTOR signaling pathway. Carcinogenesis 33: 2391-2397, 2012.

16. Zhang GJ, Xiao HX, Tian HP, Liu ZL, Xia SS and Zhou T: Upregulation of microRNA-155 promotes the migration and invasion of colorectal cancer cells through the regulation of claudin-1 expression. Int J Mol Med 31: 1375-1380, 2013.
17. Chiang Y, Song Y, Wang Z, Liu Z, Gao P, Liang J, Zhu J, Xing C and Xu H: microRNA-192, -194 and -215 are frequently downregulated in colorectal cancer. Exp Ther Med 3: 560-566, 2012.

18. Goel A and Boland CR: Recent insights into the pathogenesis of colorectal cancer. Curr Opin Gastroenterol 26: 47-52, 2010.

19. Shibuya H, Iinuma H, Shimada R, Horiuchi A and Watanabe T: Clinicopathological and prognostic value of microRNA-21 and microRNA-155 in colorectal cancer. Oncology 79: 313-320, 2010.

20. Althoff K, Lindner S, Odersky A, Mestdagh P, Beckers A, Karczewski S, Molenaar JJ, Bohrer A, Knauer S, Speleman F, et al: miR-542-3p exerts tumor suppressive functions in neuroblastoma by downregulating Survivin. Int J Cancer 136: 1308-1320, 2015.

21. Yoon S, Choi YC, Lee S, Jeong Y, Yoon J and Baek K: Induction of growth arrest by miR-542-3p that targets survivin. FEBS Lett 584: 4048-4052, 2010.

22. Condeelis J and Segall JE: Intravital imaging of cell movement in tumours. Nat Rev Cancer 3: 921-930, 2003.

23. Kedrin D, Wyckoff J, Sahai E, Condeelis J and Segall JE: Imaging tumor cell movement in vivo. Curr Protoc Cell Biol Chapter 19: Unit 19.7, 2007. doi: 10.1002/0471143030.cb1907s35.

24. Kedrin D, van Rheenen J, Hernandez L, Condeelis J and Segall JE: Cell motility and cytoskeletal regulation in invasion and metastasis. J Mammary Gland Biol Neoplasia 12: 143-152, 2007.

25. Pollard TD, Blanchoin L and Mullins RD: Molecular mechanisms controlling actin filament dynamics in nonmuscle cells. Annu Rev Biophys Biomol Struct 29: 545-576, 2000.

26. Uruno T, Liu J, Zhang P, Fan YX, Egile C, Li R, Mueller SC and Zhan X: Activation of Arp2/3 complex-mediated actin polymerization by cortactin. Nat Cell Biol 3: 259-266, 2001.

27. Hirakawa H, Shibata K and Nakayama T: Localization of cortactin is associated with colorectal cancer development. Int J Oncol 35: 1271-1276, 2009

28. Schepeler T, Reinert JT, Ostenfeld MS, Christensen LL, Silahtaroglu, Dyrskjøt L, Wiuf C, Sørensen FJ, Kruhøffer M, Laurberg S, et al: Diagnostic and prognostic microRNAs in stage II colon cancer. Cancer Res 68: 6416-6424, 2008.

29. Slaby O, Svoboda M, Fabian P, Smerdova T, Knoflickova D, Bednarikova M, Nenutil R and Vyzula R: Altered expression of miR-21, miR-31, miR-143 and miR-145 is related to clinicopathologic features of colorectal cancer. Oncology 72: 397-402, 2007.

30. Zhang L, Liu T, Huang Y and Liu J: microRNA-182 inhibits the proliferation and invasion of human lung adenocarcinoma cells through its effect on human cortical actin-associated protein. Int J Mol Med 28: 381-388, 2011.

31. Hong CC, Chen PS, Chiou J, Chiu CF, Yang CY, Hsiao M, Chang YW, Yu YH, Hung MC, Hsu NW, et al: miR326 maturation is crucial for VEGF-C-driven cortactin expression and esophageal cancer progression. Cancer Res 74: 6280-6290, 2014

32. Wang WT, Zhao YN, Yan JX, Weng MY, Wang Y, Chen YQ and Hong SJ: Differentially expressed microRNAs in the serum of cervical squamous cell carcinoma patients before and after surgery. J Hematol Oncol 7: 6, 2014.

33. Wang Y, Huang JW, Castella M, Huntsman DG and Taniguchi T: p53 is positively regulated by miR-542-3p. Cancer Res 74: 3218-3227, 2014.

34. He T, Qi F, Jia L, Wang S, Song N, Guo L, Fu Y and Luo Y: MicroRNA-542-3p inhibits tumour angiogenesis by targeting angiopoietin-2. J Pathol 232: 499-508, 2014.

35. Shen X, Si Y, Yang Z, Wang Q, Yuan J and Zhang X: MicroRNA542-3p suppresses cell growth of gastric cancer cells via targeting oncogene astrocyte-elevated gene-1. Med Oncol 32: 361, 2015. 